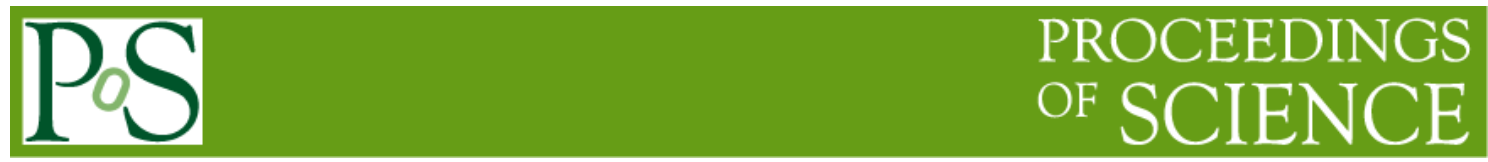

\title{
Polarized atomic beam sources: from the very start up to present days
}

\author{
D. K. Toporkov \\ Budker Institute of Nuclear Physics, \\ Novosibirsk State University, \\ Novosibirsk 630090, Russia \\ E-mail: D.K.Toporkoveinp.nsk.su
}

The history of the creation of sources of polarized particles is considered. A comparative analysis of the parameters of different sources of polarized particles used for production of polarized ions or for polarized gas targets is given. The reasons for limiting of the atomic beam intensity in such kind of sources are analyzed. The parameters of polarized gas targets obtained with the use of these sources are presented. Other sources of polarized particles are discussed.

XVth International Workshop on Polarized Sources, Targets, and Polarimetry

September 9-13, 2013

Charlottesville, Virginia, USA 


\section{Introduction}

The first quarter of the 20th century proved to be very rich in natural discoveries. This is the birth of quantum mechanics of Heisenberg and Dirac, the new quantum Bose-Einstein statistics, the birth of the Pauli exclusion principle and the hypothesis of spin. In 1925 a new fundamental concept of spin was introduced in physics by Uhlenbeck and Goudsmit [1]. It is essential that all these physicists who created the foundation of quantum mechanics were very young people. The concept of spin has proved to be very fruitful. Spin plays an important role in atomic, nuclear and particle physics and other fields. To understand the role of spin in interaction and formation of bound system such as nuclei and hadrons a lot of scattering experiments with polarized targets and polarized high energy ion or electron beams were performed. One of the intriguing and still considered one of the most important unsolved problems in physics is so called the "proton spin puzzle". The experiment carried out by the European Muon Collaboration (EMC) showed [2] that the total proton spin carried by quarks is far smaller than $100 \%$ and these results were consistent with almost zero proton spin being carried by quarks. An important question, crucial to the understanding of the internal structure of the nucleon, of how to split the total angular momentum of a gluon into spin and orbital contributions is one of the most important and interesting challenges in theory [3]. Another very interesting and important result on the distribution of charge and magnetic moment in the proton was obtained at Jlab in experiment with polarization technique [4]. The ratio of electric to magnetic form factor of the proton strongly differs from the dipole law as was measured earlier using classical Rosenbluth separation method. The radiative correction, in particular a short range two photon exchange, is likely an origin of this discrepancy [5].

In this paper we will review the gas polarized targets inserted into the storage ring of high energy charged particles - ions or electrons. The main attention will be paid to polarized hydrogen and deuterium Atomic Beam Sources (ABS) which parameters of intensity and polarization of the beam are extremely important for the quality of the internal target.

\section{Polarization of an ensembe}

The polarization of an ensemble of spin $1 / 2$ particles is described by a polarization vector. The component of polarization along some axis $(z)$ is defined as: $\mathrm{P}_{\mathrm{z}}=\mathrm{n}_{+}-\mathrm{n}_{-}$with $\mathrm{n} \pm=\mathrm{N} \pm /\left(\mathrm{N}_{+}\right.$ $\left.+\mathrm{N}_{-}\right)$, where $n_{+}$and $n_{-}$are the fractions of particles with spin I along $z\left(m_{I}=+1 / 2\right)$ and opposite to $z\left(m_{I}=-1 / 2\right)$, respectively. The polarization of deuterons ( $\left.\operatorname{spin} I=1\right)$ in an axially symmetric ensemble can be described in terms of the relative populations $n_{+}, n_{0}$ and $n_{-}$of deuterons with magnetic substates $m_{I}=+1, m_{I}=0$ and $m_{I}=-1$ with respect to the symmetry axis (direction of uniform magnetic guide field). In the absence of polarization, $n_{+}=n_{0}=n_{-}=$ $1 / 3$. The vector polarization of a deuteron target is defined as $\mathrm{P}_{\mathrm{z}}=\mathrm{n}_{+}-\mathrm{n}_{-}$, while the tensor polarization (alignment), $P_{z z}=1-3 n_{0} \quad$ describes to what extent the ensemble is depleted or enriched in particles with $m_{I}=0$.

\section{Polarized ABS}

Apparently Wrede [6], Phipps and Taylor were the first who got a polarized atomic hydrogen beam. They observed a spatial separation of atomic hydrogen beam with respect to the magnetic moment of atom in inhomogeneous magnetic field similar to well known experiment 
of Stern and Gerlach. The intensity of the beam was very low. The measured split of 0.4 $\mathrm{mm}$ between the two components of the atomic beam corresponded to the magnetic moment of atom of one Bohr Magneton. In the early $50^{\text {th }}$ of the last century Friedburg and Paul [7] suggested to apply a focusing magnet which provides more intensive atomic beam. They used six wires, placed at equal distance from the center obtaining a focusing lense similar to lenses for light. In neighboring wires current is driven in opposite direction.

Later Hamilton [8] with coworkers applied iron pole tips that significantly increased the magnetic field in the aperture. Hamilton estimated that the focusing system allows to produce a beam with the intensity of approximately two order of magnitude higher than the other system. Kantrowitz and Grey [9] suggested to use a supersonic nozzle with purpose to obtain more directed beam. Clausnitzer et al. [10] first got a polarized proton beam using an ABS with quadrupole focusing magnets. The intensity of polarized atomic beam was approximately $10^{11}$ at $/ \mathrm{sec}$. In an inhomogeneous $B$-field of multipole magnet atoms are driven towards or away from its axis, depending on the projection of the magnetic moment $\mu$ of atom at this field. For the point source of monochromatic atoms placed at the entrance of the magnet all the atoms which velocity lies inside of the solid angle $\Omega_{\mathrm{mag} .}=\pi \mu \mathrm{B}_{0} / \mathrm{kT}_{\text {nozzle }}$ may be focus to the axis. $\mathrm{B}_{0}$ is a pole tip field and $\mathrm{T}_{\text {nozzle }}$ is a temperature of atoms. In a modern ABS usually sextupole

Table 1.

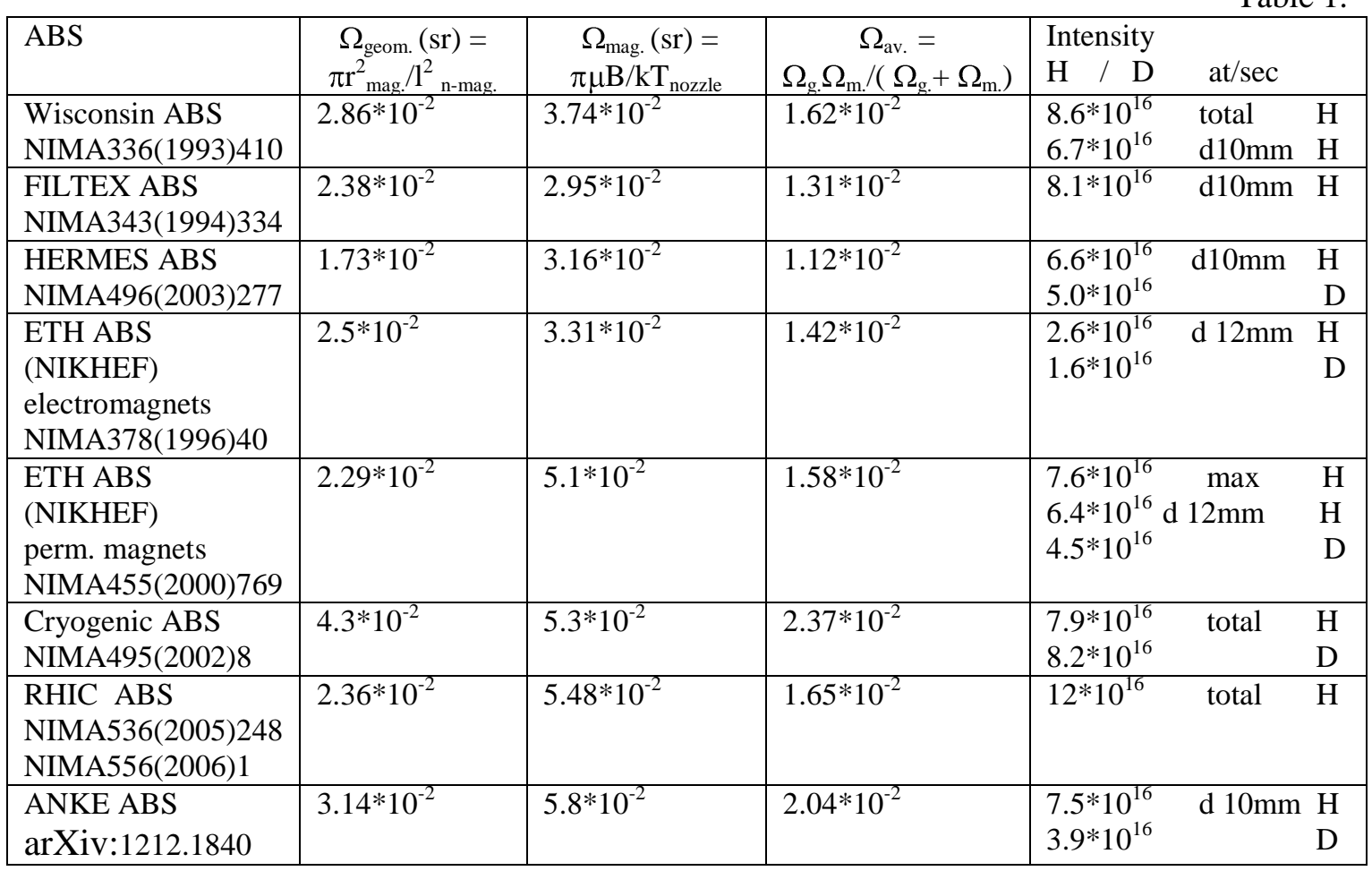

magnets are applied mostly based on permanent magnets while a superconducting or warm coil magnets are also used. The important parameter is solid angle $\Omega_{\text {geom. }}$ at which the magnet entrance is seeing from the nozzle. A product $\Omega_{\mathrm{av} .}=\Omega_{\mathrm{mag} .} * \Omega_{\mathrm{geom}} /\left(\Omega_{\mathrm{mag}}+\Omega_{\text {geom. }}\right)$ may designate the quality of ABS. Race tracking calculations are usually used to optimize the intensity from 
ABS. Table 1 presents the parameters of some ABS and intensity of a polarized beam achievable for given source. The intensities of sources are similar to each other except the RHIC ABS which demonstrate the record intensity $1.2 * 10^{17}$ at $/ \mathrm{sec}$ for hydrogen atomic beam even more than it was predicted by calculation. These ABS are used in physical experiments allowing to obtain with implementation of storage cell technique a target thickness of about $10^{14} \mathrm{at} / \mathrm{cm}^{2}$ with high polarization [11].

\section{Intensity limitation}

The intensity of polarized beam from ABS depends on many factors - flow rate of gas through the nozzle, temperature of the nozzle, degree of dissociation in the beam, focusing properties of the magnet system, vacuum conditions along the beam path. It is not easy to distinguish the influence of each factor on the resulted intensity. It is much easier to determine the influence of some factors on the intensity of molecular beam when dissociation and focusing out of the problem. Usually these measurements performed in ABS with magnets removed with the purpose to avoid additional scattering inside the magnet aperture. A lot of such measurements have been done [12]. Fig.2 presents some results on the intensity measurement of the molecular hydrogen beam at different distances from the nozzle and different nozzle temperature. One can see that the intensity of the beam measured versus the gas flow rate through the nozzle looks very similar. For large distances or low nozzle temperature the beam

\section{FILTEX ABS, 1991}

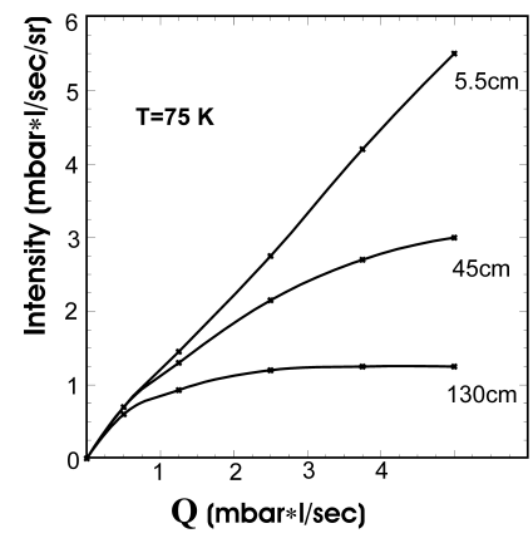

\section{T. Wise et al. NIMA 336(1993) 410}

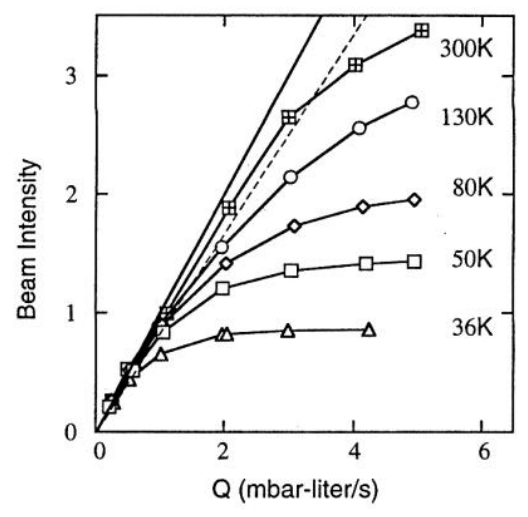

Fig.2. Intensity of the free molecular beam measured at different distances from the nozzle (left) and at fixed distance for different nozzle temperature.

intensity almost doesn't depend on the flow rate. This behavior may be explained by intrabeam scattering [13]. In this model the flux density of molecules in the parallel beam drops with distance $\mathrm{x}$ as $\mathrm{Q}(\mathrm{x})=\mathrm{Q}(0) /[1+\alpha * \mathrm{x} * \mathrm{Q}(0)]$, where $\alpha=\sigma * \Delta \mathrm{v} / \mathrm{v}^{2}, \sigma$ is a scattering cross section, $\mathrm{v}$ and $\Delta \mathrm{v}$ is a mean velocity and a width of velocity distribution in the beam. For the case of large distances, low gas velocity or high flux $\mathrm{Q}(0)$ when $\alpha * \mathrm{x} * \mathrm{Q}(0) \gg 1 \mathrm{Q}(\mathrm{x}) \sim(\alpha * \mathrm{x})^{-1}$ and doesn't depend on the initial flux $\mathrm{Q}(0)$. For the typical operating condition of modern ABS $\alpha \sim 2 * 10^{-20} \mathrm{~cm} * \mathrm{sec}$ and for $\mathrm{x}=100 \mathrm{~cm}$ one may expect the maximum flux density $\mathrm{Q}(100 \mathrm{~cm}) \sim$ $5^{*} 10^{17} / \mathrm{cm}^{2} / \mathrm{sec}$. Also the beam may become more divergent with increasing the gas flow when the freezing surface moves toward the skimmer [14]. Additional attenuation of the beam intensity may be caused by scattering of the beam particles by the residual gas along the beam path. It seems that the intensity of about $\sim 10^{17}$ at/sec is a real limit for the ABS based on Stern Gerlach separation of atoms with respect of their magnetic moment. 


\section{Summary and outlook}

The ABS discussed above were successfully used for creation of polarized hydrogen and deuterium gas targets which were applied in experiments in the storage ring of charged particles or for production of polarized ion beam. For new proposed polarized facilities [15] a filtering polarized proton target with much higher thickness is required to get a reasonable polarization time of antiproton beam. The required target thickness is more than $1 * 10^{15} \mathrm{~cm}^{-2}$. The ABS feeding storage cell method can't be used in this case. Because it is impossible to inject atomic beam into the tube where the areal density has the same order of magnitude $1 * 10^{15} \mathrm{~cm}^{-2}$, whereas the cross section for $\mathrm{H}-\mathrm{H}$ scattering is about $1 * 10^{-14} \mathrm{~cm}^{2}$. The spin-exchange optically pumped sources which have demonstrated some success in the beginning were not resulted in a reliable source of polarized atoms [16]. The recent research [17] has shown that molecules retain a high fraction of initial nuclear polarization of recombined atoms from $\mathrm{ABS}$ and that this can be used for production of more dense polarized targets. In [18] it was proposed

to use axisymmetric multipole magnet with superconducting coils to get a spatial separation of the hydrogen molecules with different projections of full moment. In such a magnet the magnetic field and its gradient are large only near the poletips. Therefore a ring aperture near the poletips can be used for a spatial separation of the substates. A capillary ring array source should provide a narrow beam of cold molecules. The geometry of the source is

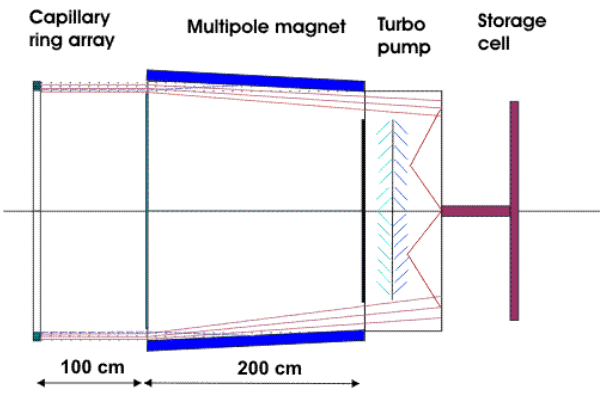

Fig.3. Suggested source of polarized molecules. presented at Fig.3. It is expected that such a source may provide the total flux of polarized molecules of about $1 * 10^{18} \mathrm{sec}^{-1}$. Preservation of nuclei polarization in a molecule during the transportation into the storage cell still remains an open question.

\section{Acknowledgments}

I am grateful to my colleagues D.M. Nikolenko, I.A. Rachek and Yu.V. Shestakov for the useful discussions and critics. In part this work was supported by the State Contract no. P522 with the Federal Agency for Education in the context of the "Scientific and ScientificEducational Personnel of Innovational Russia" Federal Goal-Oriented Program for 2009-2013.

\section{References}

[1] G. E. Uhlenbeck, S. Goudsmit, Nature 117 (1926) 264

[2] J. Ashman et al., Physics Letters B 206 (1988) 364

[3] E. Leader, C. Lorce, arXiv.org > hep-ph > arXiv:1309.4235

[4] M.K. Jones, et al., Phys. Rev. Lett. 84 (2000) 1398; A.J.R. Puckett, et al., Phys. Rev. Lett. 104 (2010) 242301

[5] P. G. Blunden, W. Melnitchouk and J. A. Tjon, Phys. Rev. Lett. 91 (2003) 142304

[6] E. Wrede, Zs. F. Physik 41 (1927) 569 
[7] H. Friedburg, W. Paul, Naturwiss 37 (1950) 20; Naturwiss 38 (1951) 159

[8] D. Hamilton, A. Lemonick, F. Pipkin, J. Reynolds, Phys. Rev. 95 (1954) 1356

[9] A. Kantrowitz, J. Grey, Rev. Sci. Instr. 22 (1951) 328

[10] G. Clausnitzer, R. Fleischman, H. Shopper, Zs. F. Physik 144 (1956) 336

[11] E.Steffens, W. Haeberli, Rep. Prog. Phys. 66 (2003) 1-49

[12] M. Stancari et al., AIP Conf. Proc. v.980 (2008) 143; N. Koch, DESY-THESIS-1999-015

[13] K. Zankel, J. Phys. B; Atom. Molec. Phys. 5 (1972) 74

[14] D.K. Toporkov, Nucl. Inst. Meth. A536 (2005) 225

[15] F. Rathmann et al., Phys. Rev. Lett. 71 (1993) 1379

[16] K.P. Coulter et al., Phys. Rev. Lett. 68 (1992) 174; Jones C E et al., Proc. 8th Int. Workshop on Polarized Sources and Targets-PST 1999 (Erlangen, 1999) 204

[17] R. Engels, this Workshop

[18] D.M. Nikolenko et al., Proc. 14th Int. Workshop on Polarized Sources, Targets and Polarimetry, PSTP 2011 (St. Petersburg, 2011) 73 